\title{
Mhicrowave Engineering
}

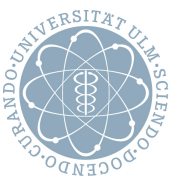

\section{UAV-Borne 2-D and 3-D Radar-Based Grid Mapping}

\author{
Philipp Hügler, Timo Grebner, Christina Knill, and Christian Waldschmidt
}

(C) 2020 IEEE. Personal use of this material is permitted. Permission from IEEE must be obtained for all other uses, in any current or future media, including reprinting/republishing this material for advertising or promotional purposes, creating new collective works, for resale or redistribution to servers or lists, or reuse of any copyrighted component of this work in other works. 


\title{
UAV-Borne 2D- and 3D-Radar Based Grid Mapping
}

\author{
Philipp Hügler, Student Member, IEEE, Timo Grebner, Christina Knill, Student Member, IEEE, \\ and Christian Waldschmidt, Senior Member, IEEE
}

\begin{abstract}
For unmanned aerial vehicles (UAVs) grid maps can be a versatile tool for navigation and self-localization. In general, payload is critical for UAVs and every additional sensor limits the flight duration. Due to its robustness and the ability to directly measure velocities, radar sensors are well suited for sense and avoid applications (SAA) for UAVs. It would be advantageous if this sensor data could be used to generated grid maps instead of mounting additional sensors like light detection and ranging (LIDAR). This letter demonstrates that using the data from high-resolution multiple-input multiple-output (MIMO) imaging radars, high-resolution 2D- and 3D-radar grid maps can be created. The necessary adaption of the sensors free-space model for MIMO radar based occupancy grid maps is presented in detail. UAV-borne measurements resulting in 2D- and 3Dgrid maps with an adequate representation of the environment validate this approach.
\end{abstract}

Index Terms-MIMO radar, frequency-modulated continuouswave radar, unmanned aerial vehicle, occupancy grid map

\section{INTRODUCTION}

In the area of robotics the problem of mapping the environment, e.g., for navigation and path planning, is studied since several decades. End of the 1980s the well-known occupancygrid that uses a probabilistic approach to represent the occupancy state of a single grid-cell based on noisy and uncertain sensor data was published [1]. Occupancy grid maps (OGMs), generated either with a known pose of the sensor or using a self localization and mapping (SLAM) algorithm [2], are nowadays heavily used. Compared to topographic or featurebased approaches, occupied and free areas can be determined directly in OGMs. Different sensor principles ranging from stereo-vision [3] over LIDAR [4] to radar sensors [5] have already been used for OGMs. Despite being quite expensive, 2D-LIDARs specifically are very popular on UAVs [4], [6] because of their high range and angular resolution. Due to the pencil beam, a single 2D-LIDAR is not enough for SAA [6] and [4] uses two 2D-LIDARs twisted by $45^{\circ}$ mounted on a rotating plate to form a 3D-LIDAR also used for mapping.

Compared to vision-based, infrared, ultrasonic or LIDAR sensors, radar sensors are very robust to environmental conditions such as rain and dust and offer high detection ranges. Their usage to generate grid maps is successfully demonstrated in the automotive field, e.g., for self-localization or autonomous path planning [7], [8]. In order to use the classical

Manuscript received December 10, 2019; accepted xxxxxxx x, xxxxx.; Date of publication Xxxxx xx, Xxxx. This work was supported by the Ministry for Science, Research, and Arts Baden-Württemberg within the project ZAFH MikroSens. (Corresponding author: Philipp Hügler.)

The authors are with the Institute of Microwave Engineering, Ulm University, 89081 Ulm, Germany (e-mail: huegler@ieee.org)

Color versions of one or more of the figures in this paper are available online at http://ieeexplore.ieee.org.

Digital Object Identifier Xx.Xxxx/XXXX.xxxx.Xxxxxxx
OGM approach for radar configurations, the sensor model has to be adjusted to the specific characteristics of the radar sensor. For UAV collision avoidance, the creation of an environmental model with $20 \mathrm{~m}$ resolution has been shown in [9] using a scanning radar. For this type of radar, e.g. with beamwidths of $1.8^{\circ}$ and $\leq 2^{\circ}$ as in [5], [10], respectively, the radar sensor model does not significantly differ from the sensor model of a LIDAR. The only extension of the model under consideration is that radar sensors are able to penetrate through vegetation and to detect objects behind other objects. However, considering state of the art MIMO imaging radar sensors, the sensor model needs further adjustments, because the field of view (FoV) of the sensors is very wide. In [7] the sensor model is improved, that the assumed free space between the radar sensor and the target uncertainty ellipsoid does not overwrite overlapping uncertainty ellipsoids of additional targets. Since higher velocities lead to higher uncertainties, [8] introduces a linear velocity dependency of the plausibility values of the uncertainty ellipsoid and a degrading factor for the free space. In addition, the complete field of view (FoV) of the radar sensor without any measured targets is declared as free space.

It has already been shown that using radar sensors on UAVs offers excellent performance for SAA [11]. This work focuses on the feasibility to use the radar data from the SAA sensor to create 2D- and 3D-radar grid maps. Due to the payload restrictions of UAVs, it is advantageous that no additional sensors are needed for high-resolution mapping. Moreover, based on the procedure in [7], the free-space model for MIMO imaging radars is improved in this letter. Instead of assuming freespace for the complete FoV, a distance and angle dependent free-space probability is introduced to account for the growing uncertainty in radar measurements with increasing distance and angle, reaching 0.5 for the maximum range and maximum FoV of the used radar sensor. Section II presents the entire signal processing chain from the radar data to the final grid map. Measurements in Section III show that high-resolution radar grid maps can be produced using the SAA sensor data and Section IV concludes this work.

\section{GRID-BASED REPRESENTATION OF RADAR DATA}

The target lists used in this work are all generated based on the same fundamental radar signal processing steps. Without loss of generality, a 2D-imaging radar with $N_{\mathrm{RX}}$ receive channels and chirp-sequence frequency modulation is used [12]. The following steps are applied to each measurement frame. For each receive channel $N_{\mathrm{R}}$ consecutive frequency ramps, each consisting of $N_{\mathrm{S}}$ time samples, are recorded:

1) Windowing: A Hann window is applied to the $N_{\mathrm{R}} \times N_{\mathrm{S}}$ time matrix in both dimensions to suppress sidelobes introduced by the successive steps. 


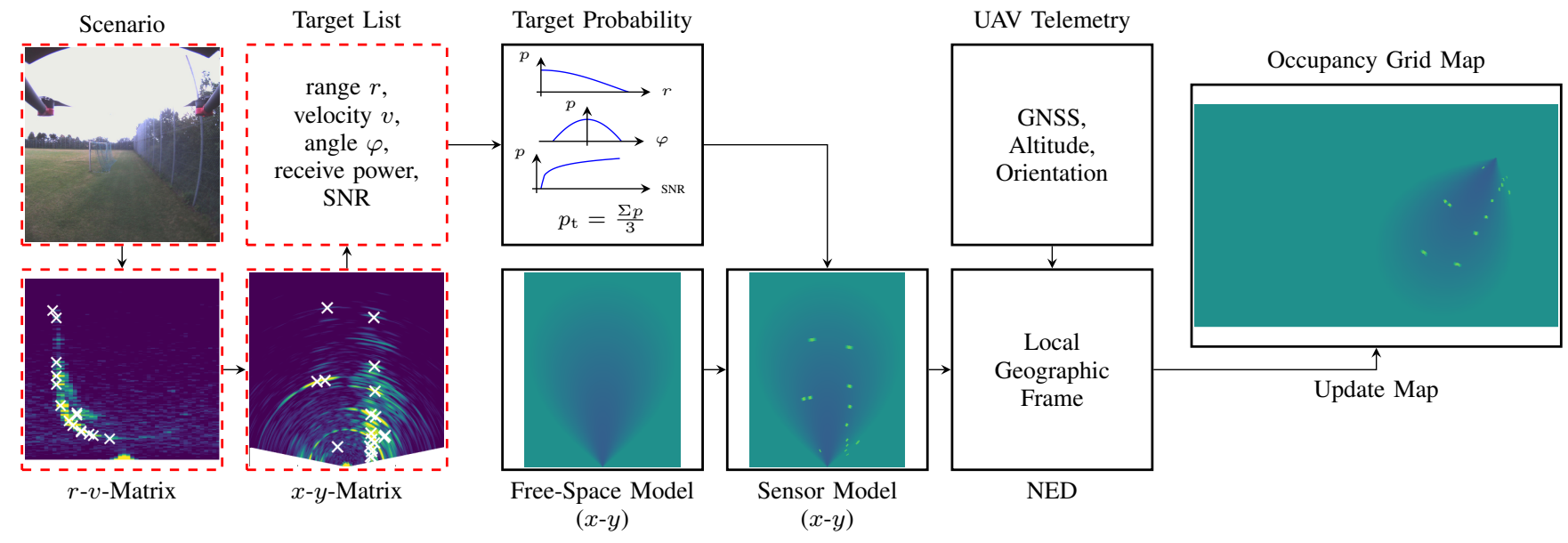

Fig. 1. Symbolic representation of the single steps from the radar measurement to the occupancy grid map. i-i-blocks describe the chirp-sequence radar processing steps for a single measurement frame with the 2D-FT range-velocity processing ( $r$ - $v$-Matrix), OS-CFAR target detection ( $\mathbf{X})$, and direction of arrival (DoA) estimation for the extracted targets ( $x-y$-Matrix), resulting in the radar target list. The target probability is calculated for the targets in the measurement frame, and all affected cells of the sensor free-space model are updated with the corresponding probability (Sensor Model). Taking into account the global position and orientation of the UAV (UAV Telemetry), the sensor model is transformed to a local geographic frame (NED, north-east-down coordinate system). Finally, all affected cells of the occupancy gird map are updated using a binary Bayes filter.

2) Range-velocity estimation: 2D-Fourier transformation (FT) of the $N_{\mathrm{R}} \times N_{\mathrm{S}}$ matrix to resolve the range-velocity $(r-v)$-matrix. The range $r$ is determined by an FT of a single ramp and the velocity $v$ by an FT of all ramps of each range cell.

3) Accumulation: All $N_{\mathrm{RX}} r-v$-matrices of the receivers are accumulated into a single $r$ - $v$-matrix by a non-coherent integration.

4) Target extraction: An ordered statistic constant false alarm rate (OS-CFAR) algorithm [13] is separately applied in the $r$ and $v$ dimension. A target is only valid if it is detected in both dimensions.

5) Direction of arrival (DoA) estimation: For each extracted target the azimuth angle $\varphi$ is determined using an FT along all receiving channels.

6) Target list: A target list containing $r, v, \varphi$, the receive power and the SNR of the targets is created.

Steps 3) to 6) are exemplified by the '-blocks in Fig. 1. The $x-y$-matrix is only used to visualize the DoA estimation. In addition to the radar target list, the global UAV position and orientation (quaternions) for each measurement frame is needed to map the detected targets to a local geographic frame represented by a north-east-down (NED) coordinate system. In the following, the basics of the two grid-based representations used in this paper are introduced.

\section{A. Amplitude Grid Map}

An easy and computationally fast approach for a grid-based representation of radar data is the amplitude grid map (AGM) proposed in [7]. Assuming point-like targets, the free-space losses are compensated by raising the target receive power by $40 \mathrm{~dB}$ per range decade. At the cost of reduced positional accuracy, the AGM reduces the radar measurement uncertainty by calculating the mean of the complete measurement time of the weighted target amplitude $\left(\propto \frac{1}{r}\right)$ affecting the grid cell under consideration.

\section{B. Occupancy Grid Map}

In this form of representation the map is build up as a grid, with each cell storing a value representing its occupancy probability (between 0: free space and 1: fully occupied). An in-depth derivation can be found in [2]. In general, a sensor model is used to update the probability of all cells within the sensors FoV. This model depends strongly on the type of sensor (e.g. LIDAR, sonar, or radar) and therefore, the implementation for MIMO imaging radars is described in detail in this work. It is taken into account that for example in contrast to LIDAR, a radar can sense targets, which are optically hidden behind other targets. The sensor model is formed by a free-space model describing the FoV of the radar sensor. This model is then updated with the targets found by the radar sensor.

The probability of the free-space model $\left(p_{\mathrm{FSM}}\right)$ depends on the maximum range $r_{\max }$ and the $\mathrm{FoV}$ of the radar sensor. Since the same target is not measured at the exact same position in each radar measurement, the minimum of $p_{\mathrm{FSM}}$ is set to 0.3 in order to prevent a too strong neutralization of previous measurements. In range, the plausibility degrades $\propto \frac{1}{r^{2}}$ from 1.0 until it reaches 0.5 for $r_{\max }$. In azimuth, the FoV is characterized by the directivity of a single channel (in this work the directivity of a single microstrip patch antenna), and is approximated by a cosine function, which reaches 0.5 at the maximum FoV. The sum of both results in

$$
\begin{aligned}
p_{\mathrm{FSM}}(r, \varphi)=2.3 & -\exp \left(\frac{\ln (1.2-0.5)}{r_{\max }^{2}} r^{2}\right) \\
& -\cos \left(\frac{\operatorname{acos}(0.5)}{\mathrm{FoV}} \varphi\right) .
\end{aligned}
$$

The addend 2.3 sets the minimum of the plausibility to 0.3 . In addition, the plausibility needs to be limited to 0.5 :

$$
p_{\mathrm{FSM}}(r, \varphi)=\max \left(p_{\mathrm{FSM}}(r, \varphi), 0.5\right) .
$$

The resulting free-space model can be seen in Fig. 1. 
For each measurement frame, the free-space model is updated with the targets provided by the target list. Target plausibility $p_{\mathrm{t}}$ consists of individual plausibility measures for range $p_{\mathrm{r}}$, azimuth $p_{\varphi}$, and $\mathrm{SNR} p_{\mathrm{SNR}}$ according to

$$
\begin{aligned}
p_{\mathrm{r}} & =\exp \left(\ln \left(\frac{0.5}{r_{\max }^{2}}\right) r^{2}\right), \\
p_{\varphi} & =\cos \left(\frac{\operatorname{acos}(0.5)}{\mathrm{FoV}} \varphi\right), \\
p_{\mathrm{SNR}} & =1-\exp \left(\left.a \mathrm{SNR}\right|_{\mathrm{dB}}+b\right)^{-1},
\end{aligned}
$$

where the scaling factor $a$ and offset $b$ for $p_{\mathrm{SNR}}$ are determined by solving (5) for the minimum and maximum reasonable SNR of the radar sensor for the probabilities 0.5 and 1 , respectively. Each single plausibility is limited to a minimum of 0.5 , and $p_{\mathrm{t}}$ is calculated by taking the mean of the probabilities

$$
p_{\mathrm{t}}=\frac{p_{\mathrm{r}}+p_{\varphi}+p_{\mathrm{SNR}}}{3} .
$$

In order to obtain the sensor model, in addition to the target probability the measurement uncertainties in range and azimuth are considered. The standard deviation in range $\sigma_{\mathrm{r}}$ for frequency modulated continuous wave (FMCW) radars with bandwidth $B$ and in azimuth $\sigma_{\varphi}$ with the $3 \mathrm{~dB}$ beamwidth $\Delta \varphi_{3 \mathrm{~dB}}$ are stated in [14] and [15] respectively:

$$
\sigma_{\mathrm{r}} \geq \sqrt{\frac{3 c_{0}^{2}}{(2 \pi)^{2} \mathrm{SNR} N_{\mathrm{S}} B^{2}}} \quad \text { and } \quad \sigma_{\varphi}=\frac{\Delta \varphi_{3 \mathrm{~dB}}}{1.6 \sqrt{2 \mathrm{SNR}}} .
$$

For an SNR of $10 \mathrm{~dB}$ and with the values stated in Table I it can be deduced that $\sigma_{\mathrm{r}}$ is neglectable because it results in sub-millimeter accuracy and is much smaller then the range resolution (centimeter range). With the target probability $p_{\mathrm{t}}$ and $\sigma_{\varphi}$ the free-space model is updated assuming a Gaussian distribution. All cells in radial distance $r$ and in the affected angular range $\varphi \pm 3 \sigma_{\varphi}$ are overwritten if the probability value is greater than the probability of the free-space model at these cells. This procedure ensures that already inserted targets are not overwritten by weaker targets.

After transforming the sensor model into the local NED coordinate system, the occupancy map is updated using a binary Bayes filter [2].

The previously described procedure can easily be extended for $3 \mathrm{D}$-grid maps by taking into account the elevation angle $\vartheta$. Depending on the used antenna, a third subtractive term can be added to equation (1). The same applies for the update process in the sensor model. Beside the additional term for the probability in elevation $p_{\vartheta}$ for the overall target probability, a 2D-Gaussian distribution with standard deviation $\sigma_{\vartheta}$ for the elevation angle is used to calculate the probability of the affected cells $\left(r, \varphi \pm 3 \sigma_{\varphi}\right.$, and $\left.\vartheta \pm 3 \sigma_{\vartheta}\right)$.

\section{MEASUREMENT RESUlts}

The measurement system is based on a $77 \mathrm{GHz}$ timedivision multiplexing (TDM) high-resolution MIMO imaging radar with exchangeable antenna front-end. By replacing the antenna array, the same system can be used for 2D- and 3Dimaging. The radar and the measurement PC are mounted to a hexacopter that can lift up to $6 \mathrm{~kg}$ with a flight time of
TABLE I

USED RADAR PARAMETERS FOR GRID MAP REPRESENTATIONS.

\begin{tabular}{l|c|c|c} 
Parameter & 2D & 2.5D & 3D \\
\hline Bandwidth $B$ & \multicolumn{3}{|c}{$2 \mathrm{GHz}$} \\
Ramp time $T_{\mathrm{R}}$ / repetition time $T_{\mathrm{RRI}}$ & \multicolumn{3}{|c}{$50 \mathrm{\mu s} / 60 \mathrm{\mu s}$} \\
Sampling frequency $f_{\mathrm{S}}$ & \multicolumn{3}{|c}{$20 \mathrm{MHz}$} \\
Measurement rate & \multicolumn{2}{|c}{$15 \mathrm{~Hz}$} \\
Number of receivers $N_{\mathrm{RX}}$ & \multicolumn{2}{|c}{8} \\
\hline Number of transmitters $N_{\mathrm{TX}}$ & \multicolumn{2}{|c|}{3} & 4 \\
Number of ramps $N_{\mathrm{R}}$ & \multicolumn{2}{|c|}{258} & 256 \\
FoV azimuth & \multicolumn{2}{|c|}{ $\pm 80^{\circ}$} & $\pm 60^{\circ}$ \\
\hline FoV elevation & - & $\pm 3.5^{\circ}$ & $\pm 30^{\circ}$
\end{tabular}

approximately $15 \mathrm{~min}$. All measurement data (radar data, camera image, UAV telemetry) is stored by the measurement PC, synchronized by a GNSS timestamp, and processed offline. An in-depth description of the complete measurement system is given in [11]. The most important radar parameters are listed in Table I. They result in a range resolution of $75 \mathrm{~mm}$ and a maximum range of $37 \mathrm{~m}$. The angular resolution in azimuth for the $2 \mathrm{D} / 2.5 \mathrm{D}$-case is $3^{\circ}$ and for the $3 \mathrm{D}$-case $10^{\circ}$ in azimuth and $23^{\circ}$ in elevation. In the following, the gridmap approches discussed in Section II are applied to different scenarios. For all measurements, the probability saturation of the OGM is set to 0.1 and 0.9 .

\section{A. Soccer Pitch - A 2D-Representation}

To investigate the difference between the two described grid map approaches a soccer pitch partly bordered by a high fence is chosen as the first measurement scenario. A $3 \times 8 \mathrm{MIMO}$ antenna system is applied, yielding a 24-element uniform linear array (ULA) facing forward in flight direction. The $\mathrm{UAV}$ is manually maneuvered around the soccer pitch with an approximate altitude above ground level of $2 \mathrm{~m}$. During the three minute flight 2650 radar measurements were recorded.

The results of the AGM and OGM with a resolution of $0.1 \mathrm{~m}$ are shown in Fig. 2 (a) and (b), respectively. In each of the two maps, the fence of the soccer pitch, the soccer goals and the open areas can clearly be identified. Even reflections caused by undergrowth behind the fence are included in both maps. Using the OGM results in a much sharper representation overall. Looking at the magnified area (c) the free-space between the goal posts and the fence can be identified much clearer compared to the AGM.

A problematic region in the OGM is identified in scope (d). While the right-angled fence corner can clearly be recognized in the AGM, a very low occupancy value is present in the OGM. Since the UAV flies straight ahead towards the fence, the fence occurs as an extended target with multiple reflections in the same range-velocity cell. No multi-target estimation is performed in the angular domain, therefore, only the most dominant reflection is considered, which occurs under a different angle each radar frame. As a consequence, the occupancy likelihood is decreased frame by frame, leading to this unfavorable partial result. However, scope (e) highlights the much better resolution of the OGM. The goal next to the fence is only clearly distinguishable in the OGM. For a better impression of the soccer pitch, Fig. 3 depicts the OGM superimposed with a satellite image. 


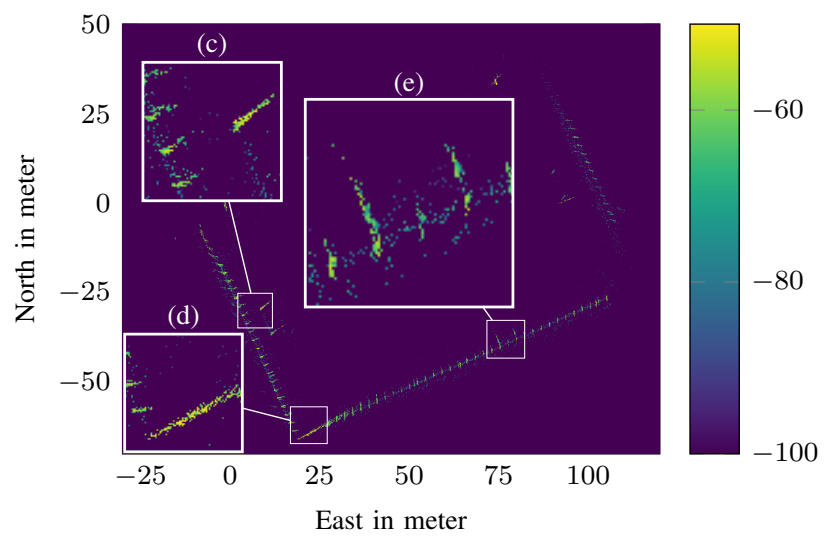

(a)

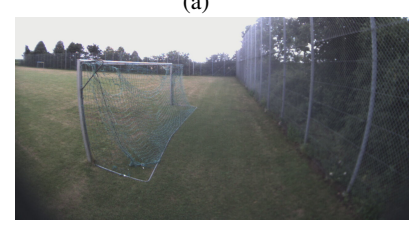

(c)

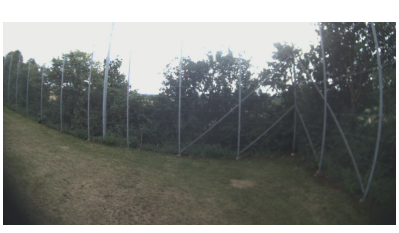

(d)

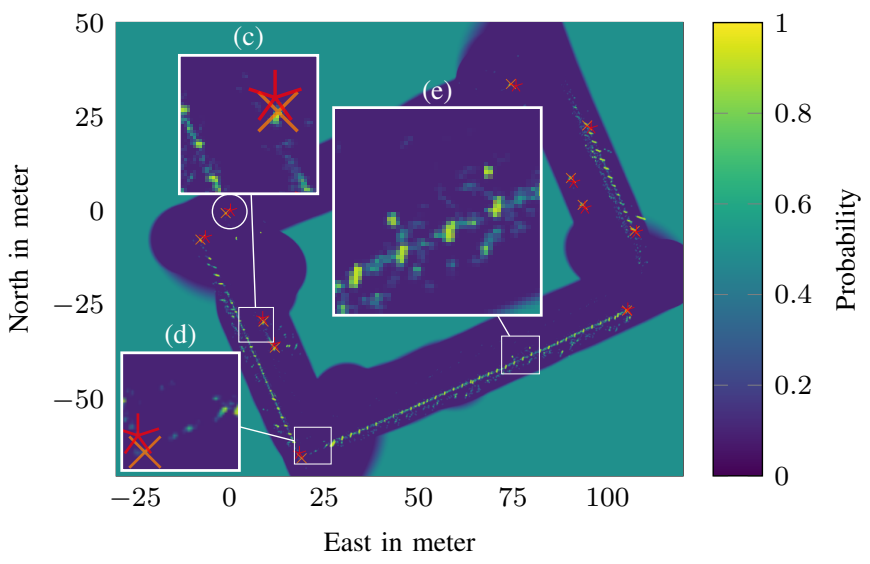

(b)

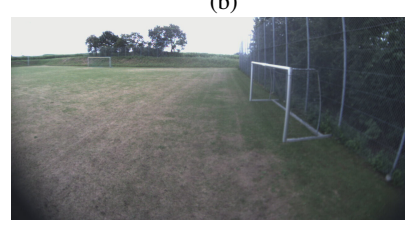

(e)

Fig. 2. Measurement results of a soccer pitch (a) amplitude and (b) occupancy grid map with a grid resolution of 0.1 m. In (c), (d), and (e) images taken by the UAV camera of interesting points highlighted in the two different grid map approaches are displayed. 12 distinctive points such as lamp- and goalposts, as well as the start- and endpoints of the fences are marked with $(\star)$ using a tachymeter and with $(\times)$ extracted from the OGM.

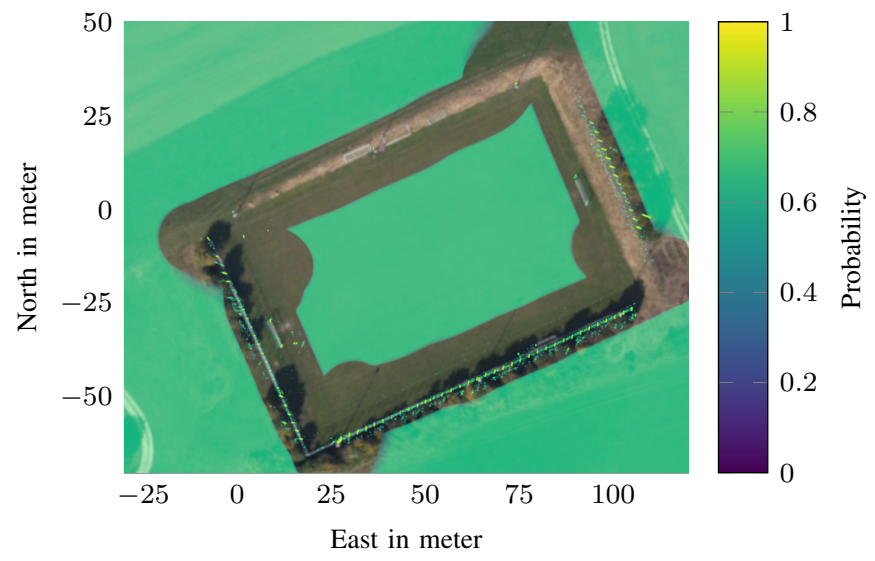

Fig. 3. The satellite image of the soccer pitch superimposed with the occupancy grid map. Satellite image: Google, GeoBasis-DE/BKG.

For ground truth data 12 distinct points of the soccer pitch have been measured using a tachymeter and are marked with (*) in Fig. 2(b). The same points have been extracted from the OGM and are marked with $(\times)$. The mean displacement of the $(\star)$-points to the $(\times)$-points amounts to $1.14 \mathrm{~m}$, which is due to the drift of the GPS. More meaningful is the distances between the points relative to the encircled point in the corresponding domain. Calculating the standard deviation of the discrepancy between the distances of $(\star)$ and $(x)$ results in $0.48 \mathrm{~m}$. Additionally, the position of the UAV has also been tracked using the tachymeter. Applying the same analysis to the resulting OGM with the tachymeter-based positions leads to a mean displacement of $0.30 \mathrm{~m}$, and the standard deviation of the discrepancy between the distances amounts to $0.13 \mathrm{~m}$.

\section{B. Stone Wall - A 2.5D-Representation}

In order to generate a 3D-terrain model without the usage of a 3D-imaging radar, a 2D-imaging radar in combination with the movement of the UAV can be used. For this purpose, the already mentioned 24-element ULA is facing downwards and the UAV is flying along a stepped stone wall depicted in Fig. 4(a). Because no DoA estimation is performed in the elevation-axis, we call this approach $2.5 \mathrm{D}$, nevertheless it results in a 3D-OGM. The free-space model is adjusted to the $3 \mathrm{D}$-scenario following the procedure described in Section II-B, and the resulting beam for probabilities smaller 0.5 is depicted in Fig. 4 (b). Displaying all occupied cells with a probability greater 0.65 , the OGM with a grid resolution of $0.2 \mathrm{~m}$ of the stepped stone wall depicted in Fig. 4 (c) is obtained. Beside some clutter caused by bushes, the distinct levels can clearly be identified and the extracted height ranges between $1.0 \mathrm{~m}$ to $1.4 \mathrm{~m}$. This is in good consistency with the overall height of the five steps, each with a height between $0.20 \mathrm{~m}$ to $0.25 \mathrm{~m}$.

\section{University Campus - A 3D-Representation}

To evaluate the procedure in a more complex area, the UAV flies through a passage with a footbridge. A 3D-imaging radar was used with a 32-element 2D-quasi-ULA to generate a 3DOGM. The antenna positions of the used array are depicted in Fig. 5(a). Fig. 5(b) shows a picture of the passage on the campus with the footbridge and trees. A section of the resulting 3D-OGM representing the footbridge is depitcted in (c). The free space below the footbridge can clearly be identified in the OGM and matches the real dimensions with a height of about $5 \mathrm{~m}$ and a width of around $8 \mathrm{~m}$. 


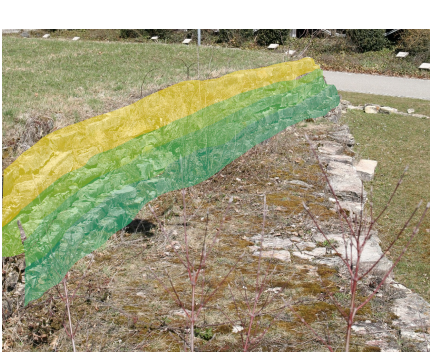

(a)

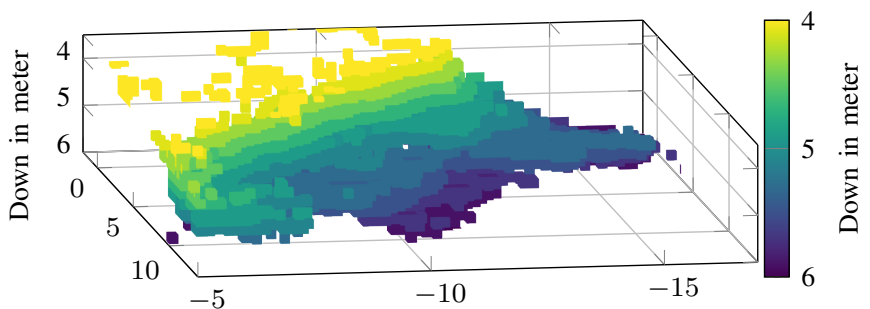

North in meter

East in meter

(c)

Fig. 4. The stepped stone wall with a step height of $0.20 \mathrm{~m}$ to $0.25 \mathrm{~m}$ in (a) is mapped using a $2 \mathrm{D}$-imaging radar. The beam of the free-space model for probabilities smaller 0.5 is depicted in (b). (c) shows the result for a probability greater 0.65 of the $2.5 \mathrm{D}$-occupancy grid map with a grid resolution of $0.2 \mathrm{~m}$.

\section{CONCLUSION}

In this paper the potential of creating 2D- and 3D-OGMs for an UAV-borne high-resolution MIMO imaging radar has been successfully demonstrated. The derived 2D-OGM using telemetry-based positions features high resolution, and the relative distances between extracted reference points show only a standard deviation of $0.48 \mathrm{~m}$ compared to the real distances. Using tachymeter-based positions reduces the standard deviation to $0.13 \mathrm{~m}$, which is in the same order as the grid size. Thus, the accuracy of the OGM is mainly limited by the positioning errors. Utilizing the degrees of freedom of the $\mathrm{UAV}$, terrain profiles represented by 3D-OGMs can be created despite using a 2D-imaging radar. Finally, a 3D-OGM of a passage has been recorded with a 3D-imaging radar. In this map, a footbridge connecting two buildings can clearly be identified as traversable.

\section{REFERENCES}

[1] A. Elfes, "Using Occupancy Grids for Mobile Robot Perception and Navigation," Computer, vol. 22, no. 6, pp. 46-57, Jun. 1989.

[2] S. V. Thrun, W. V. Burgard, and D. V. Fox, Eds., Probabilistic robotics, ser. Intelligent robotics and autonomous agents. Cambridge, Mass.; London: MIT Press, 2005.

[3] C. Yu, V. Cherfaoui, and P. Bonnifait, "Evidential Occupancy Grid Mapping with Stereo-vision," in IEEE Intelligent Vehicles Symposium (IV), Jun. 2015, pp. 712-717.

[4] M. Beul, N. Krombach, Y. Zhong, D. Droeschel, M. Nieuwenhuisen, and S. Behnke, "A High-performance MAV for Autonomous Navigation in Complex 3D Environments," in International Conference on Unmanned Aircraft Systems (ICUAS), Jun. 2015, pp. 1241-1250.

[5] M. Adams, J. Mullane, E. Jose, and B.-N. Vo, Robotic Navigation and Mapping with Radar. Artech House, 2012.

[6] M. ki, J. cha, and H. Lyu, "Detect and avoid system based on multi sensor fusion for UAV," in International Conference on Information and Communication Technology Convergence (ICTC), Oct. 2018, pp. $1107-1109$.

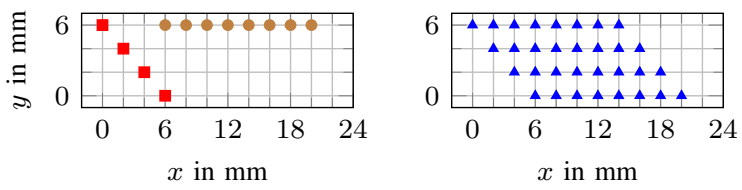

(a)

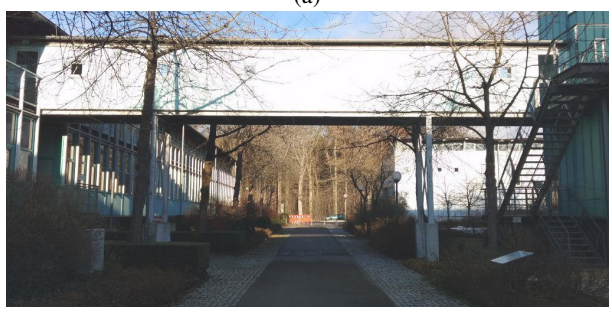

(b)
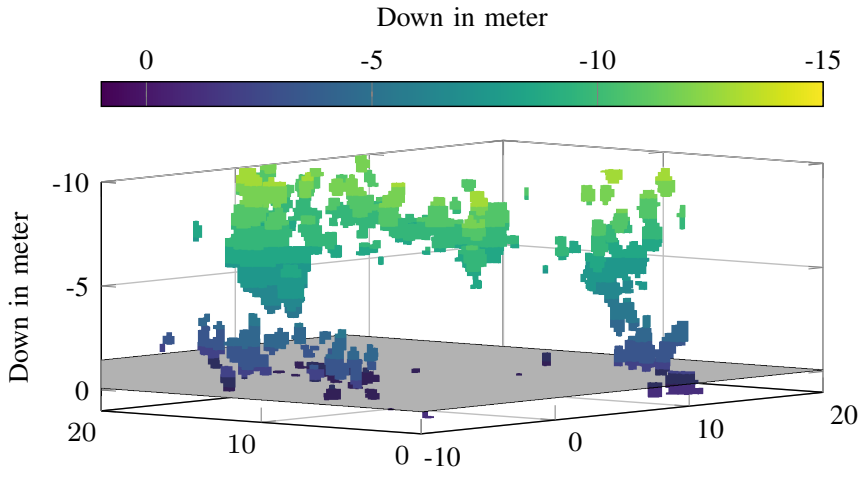

North in meter

East in meter

(c)

Fig. 5. The antenna positions of the used 32-element 2D-quasi-ULA are depicted in (a), with receiver, $\square$ transmitter, and $\Delta$ virtual element positions. (b) shows an image of a passage with a footbridge between two buildings. For a probability greater 0.65 the $3 \mathrm{D}-\mathrm{OGM}$ of the footbridge is given in (c).

[7] K. Werber, M. Rapp, J. Klappstein, M. Hahn, J. Dickmann, K. Dietmayer, and C. Waldschmidt, "Automotive Radar Gridmap Representations," in IEEE MTT-S International Conference on Microwaves for Intelligent Mobility (ICMIM), Apr. 2015, pp. 1-4.

[8] R. Prophet, H. Stark, M. Hoffmann, C. Sturm, and M. Vossiek, "Adaptions for Automotive Radar Based Occupancy Gridmaps," in IEEE MTT-S International Conference on Microwaves for Intelligent Mobility (ICMIM), Apr. 2018, pp. 1-4.

[9] Z. Szymański, S. Jankowski, and J. Szczyrek, "Reconstruction of environment model by using radar vector field histograms," in Photonics Applications in Astronomy, Communications, Industry, and High-Energy Physics Experiments, vol. 8454. SPIE, 2012, pp. $611-618$.

[10] A. Foessel, J. Bares, and W. R. L. Whittaker, "Three-Dimensional Map Building with MMW Radar," in Proceedings of the 3rd International Conference on Field and Service Robotics, R. C. A. Halme and E. Prassler, Eds. Helsinki, Finland: Yleissjhaljennhos-Painophorssi, Jun. 2001.

[11] P. Hügler, F. Roos, M. Schartel, M. Geiger, and C. Waldschmidt, "Radar Taking Off: New Capabilities for UAVs," IEEE Microwave Magazine, vol. 19, no. 7, pp. 43-53, Nov. 2018.

[12] V. Winkler, "Range Doppler detection for automotive FMCW Radars," in European Radar Conference (EuRAD), Oct. 2007, pp. 166-169.

[13] H. Rohling, "Radar CFAR Thresholding in Clutter and Multiple Target Situations," IEEE Transactions on Aerospace and Electronic Systems, vol. AES-19, no. 4, pp. 608-621, Jul. 1983.

[14] S. Scherr, R. Afroz, S. Ayhan, S. Thomas, T. Jaeschke, S. Marahrens, A. Bhutani, M. Pauli, N. Pohl, and T. Zwick, "Influence of Radar Targets on the Accuracy of FMCW Radar Distance Measurements," IEEE Transactions on Microwave Theory and Techniques, vol. 65, no. 10, pp. 3640-3647, Oct. 2017.

[15] G. R. V. Curry, Ed., RADAR SYSTEM PERFORMANCE MODELING, 2nd ed., ser. Artech House radar library. Boston, Mass. ; London: Artech House, 2005. 